\title{
Nutritional Screening and Clinical Outcome in Hospitalized Patients with Crohn's Disease
}

\author{
Azusa Takaoka ${ }^{a}$ Masaya Sasaki ${ }^{a}$ Naoko Nakanishia Mika Kurihara ${ }^{a}$ \\ Akiko Ohi $^{a}$ Shigeki Bamba ${ }^{a}$ Akira Andoh $^{b}$ \\ a Division of Clinical Nutrition, Shiga University of Medical Science, Otsu, Japan; ${ }^{b}$ Department of Gastroenterology, \\ Shiga University of Medical Science, Otsu, Japan
}

\section{Keywords}

Crohn's disease · Subjective Global Assessment · Nutritional Risk Screening $2002 \cdot$ Serum albumin

\begin{abstract}
Background/Aims: Hospitalized patients with Crohn's disease (CD) can develop severe nutritional deficits. However, the nutritional screening tools with the most utility for such patients are still unknown. Methods: Nutritional status of 40 $\mathrm{CD}$ patients was assessed on admission using several screening tools and laboratory tests. Their validity was evaluated in relation to length of hospital stay (LOS) and intestinal resection. Receiver operating characteristic analysis was performed to predict prolonged LOS ( $\geq 28$ days). Results: Prolonged LOS was correlated with each of the following screening parameters: Subjective Global Assessment, Nutrition Risk Screening 2002 (NRS 2002), Onodera's Prognostic Nutritional Index (O-PNI), Controlling Nutritional Status, serum albumin level, and weight loss. These parameters were not correlated with intestinal resection. Evaluation of prognostic yield showed cutoff values of serum albumin $3.3 \mathrm{~g} / \mathrm{dL}$ (AUC 0.797 , sensitivity $57.1 \%$, specificity $89.5 \%$ ) and O-PNI 36.5 $(0.749,71.4 \%, 73.7 \%)$. By combining the serum albumin cut-
\end{abstract}

\section{KARGER}

(๑) 2017 S. Karger AG, Basel

E-Mail karger@karger.com

www.karger.com/anm off value and NRS 2002 score, patients were divided into 4 groups, with a prolonged LOS rate of $68.2 \%$ in the group with the worst prognosis. Conclusions: A combination of serum albumin (given the simplicity of testing) and NRS 2002 as nutritional screening tools may be useful for hospitalized $C D$ patients.

(c) 2017 S. Karger AG, Basel

\section{Introduction}

Crohn's disease (CD) is a chronic inflammatory disorder of the digestive tract of unknown etiology. Genetic and environmental factors have been associated with the pathogenesis of CD [1-3], but the precise mechanisms remain unclear. Clinical features of CD include diarrhea, abdominal pain, weight loss, and other abdominal symptoms due to bowel obstruction. Patients also have various nutritional and metabolic disturbances. Among hospitalized CD patients, $20-70 \%$ show emaciation $[4,5]$ and approximately $75 \%$ are undernourished $[6,7]$. Furthermore, a reported $41 \%$ of hospitalized CD patients were evaluated as being severely malnourished based on the Subjective Global Assessment (SGA) of nutritional status
Masaya Sasaki, MD, $\mathrm{PhD}$

Division of Clinical Nutrition Shiga University of Medical Science

Seta-Tsukinowa, Otsu, Shiga 520-2192 (Japan)

E-Mail sasaki@belle.shiga-med.ac.jp 
[8]. Nutritional support is therefore essential for CD patients.

At admission, it is important to evaluate the nutritional status of $C D$ patients because instituting nutritional intervention early is essential, particularly for malnourished patients. However, there is no standard tool for evaluating nutritional status in CD patients. Various nutritional screening tools have been developed, including the SGA, Malnutrition Universal Screening Tool(MUST), and Nutritional Risk Screening 2002 (NRS 2002). The SGA was developed in $1982[9,10]$, and in 2004, the SGA score was reported to be correlated separately with serum albumin level, total lymphocyte count, and total cholesterol level [11]. The European Society for Clinical Nutrition and Metabolism guidelines [12] recommend the MUST [13] for nutritional evaluation of adults in the community and the NRS 2002 [14] for detection of undernutrition and the risk of its development in hospital settings. In addition, Onodera's Prognostic Nutritional Index (O-PNI) and Controlling Nutritional Status (CONUT) are nutritional screening tools used as objective nutritional indexes. The validity of the O-PNI, which comprises serum albumin level and total lymphocyte count, was first reported to predict prognosis in 189 gastrointestinal surgical patients [15]. The CONUT was originally reported as a screening tool to assess nutritional status, and its score consists of 3 parameters - serum albumin, cholesterol, and total lymphocytes - each of which can be easily calculated [16].

These nutritional screening tools have been reported to be useful for predicting prognosis in conditions such as chronic kidney disease [17] and chronic heart failure [18]. To our knowledge, the present study is the first to report a relationship between nutritional screening results and prognosis of hospitalized patients with CD.

\section{Patients and Methods}

\section{Patients}

A total of 40 patients with CD who were admitted to the Gastroenterology Unit of Shiga University of Medical Science Hospital between June 2011 and July 2016 were enrolled this study. The Ethics Committee of Shiga University of Medical Science approved this study. All diagnoses of CD were established using radiological, endoscopic, histological, and clinical criteria.

\section{Methods}

The following values were measured on admission: (1) anthropometrics - height $(\mathrm{cm})$, weight $(\mathrm{kg})$, body mass index (BMI; kg/ $\mathrm{m}^{2}$ ); (2) nutritional screening tools - SGA [9, 10], MUST [13], NRS 2002 [14], O-PNI [15], and CONUT [16]; (3) laboratory tests - levels of total protein $(\mathrm{g} / \mathrm{dL})$, albumin $(\mathrm{g} / \mathrm{dL})$, total cholesterol $(\mathrm{mg} / \mathrm{dL})$, triglycerides $(\mathrm{mg} / \mathrm{dL})$, hemoglobin $(\mathrm{g} / \mathrm{dL})$, C-reactive protein $(\mathrm{mg} / \mathrm{dL})$, and total lymphocyte count (cells $\left./ \mathrm{mm}^{3}\right)$; and (4) activity index - Crohn's Disease Activity Index (CDAI) [19, 20]. The validity of these screening tools was evaluated separately in relation to intestinal resection and length of hospital stay (LOS).

\section{Nutritional Screening Tools}

The SGA questionnaire covers the patient's history and physical examination. History includes weight loss, changes in food intake, gastrointestinal symptoms, functional capacity, and underlying disease. Physical examination includes muscle mass, subcutaneous fat, presence of edema and ascites, and the clinician's overall assessment of the patient's status. Patients are classified as well nourished, moderately malnourished, or severely malnourished $[9,10]$.

The MUST evaluates 3 independent criteria: BMI, loss of body weight, and acute disease-effect score. Patients are classified by the total of each score as low risk, medium risk, or high risk [13].

The NRS 2002 consists of an initial and a final screening. The final screening involves completing a questionnaire covering nutritional score (loss of body weight, BMI, and food intake), severity of disease score, and age adjustment for patients aged $>70$ years. Patients are classified based on the total of each score as being with or without nutritional risk [14].

The O-PNI is calculated as $10 \times$ albumin $(\mathrm{g} / \mathrm{dL})+0.005 \times$ total lymphocyte count per cubic millimeter [15]. The CONUT assigns scores for serum albumin, total lymphocyte count, and total cholesterol [16].

\section{Statistical Analyses}

Statistical analyses were performed using the chi-square test when appropriate. Correlation was investigated using Spearman rank correlation tests. Receiver operating characteristic (ROC) curves were plotted to compare the capability of each clinical variable to predict prolonged LOS ( $\geq 28$ days). Results are presented as median $(25-75 \%)$, and a $p$ value of $<0.05$ was considered statistically significant. All statistical analyses were performed using SPSS version 22 (SPSS, Inc., Chicago, IL, USA).

\section{Results}

\section{Basic Patient Characteristics}

Table 1 shows the patient characteristics. Of the total 40 patients, 17 patients $(42.5 \%)$ had mild disease (CDAI $<220)$ and 23 patients $(57.5 \%)$ had moderate to severe disease (CDAI $\geq 220)$. It was shown that 14 patients (35\%) had BMI $<18.5 \mathrm{~kg} / \mathrm{m}^{2}$. The reasons for admission were as follows: exacerbation of disease, 16; abscess formation, 14; ileus, 7; and bleeding, 3 .

\section{Prevalence of Malnutrition}

Based on the SGA as a screening tool for malnutrition, $37.5 \%$ of patients were categorized as being severely malnourished. Based on the MUST, however, $60 \%$ of 
Table 1. Clinical background of the patients $(n=40)$

\begin{tabular}{lc}
\hline Characteristics & CD patients $(n=40)$ \\
\hline Gender, male/female & $30 / 10$ \\
Age, years & $32.4(25.3-37.8)$ \\
Height, cm & $168.7(162.6-173.5)$ \\
Body weight, kg & $55.2(50.4-59.8)$ \\
BMI, kg/m ${ }^{2}$ & $19.2(17.6-22.3)$ \\
Type of CD (ileal/colonic/ileocolonic) & $17 / 4 / 19$ \\
Activity index (CDAI) & $228.1(166.9-308.3)$ \\
Treatments & \\
$\quad$ PSL, mg/day & \\
$\quad \geq 20$ & 9 \\
$\quad<20$ & 3 \\
$\quad$ Azathioprine & 15 \\
$\quad$ Anti-TNF- $\alpha$ & 30 \\
Nutritional therapy & \\
TPN/PPN & $23 / 14$ \\
EN (switched from PN) & 39 \\
Intestinal resection & 13 \\
LOS, days & $25.5(13.5-45.0)$ \\
Laboratory tests & \\
Total protein, g/dL & $6.4(5.8-6.8)$ \\
Serum albumin, g/dL & $3.0(2.7-3.4)$ \\
Total cholesterol, mg/dL & $133.0(120.0-161.3)$ \\
Triglyceride, mg/dL & $89.0(67.5-116.0)$ \\
Hemoglobin, g/dL & $11.5(9.3-13.0)$ \\
C-reactive protein, mg/dL & $3.4(0.5-10.0)$ \\
\hline
\end{tabular}

Date were presented as median (25-75\%).

$\mathrm{CD}$, Crohn's disease; BMI, body mass index; CDAI, Crohn's Disease Activity Index; PSL, prednisolone; anti-TNF- $\alpha$, anti-tumor necrosis factor- $\alpha$; TPN, total parenteral nutrition; PPN, peripheral parenteral nutrition; EN, enteral nutrition; $\mathrm{PN}$, parenteral nutrition; LOS, length of stay in the hospital.

patients were classified as being at high risk of malnutrition. According to the NRS 2002, 67.5\% of patients were classified to be at risk, almost the same frequency as determined by the MUST. Loss of body weight over a 6-month period was $6.1 \%$ (2.3-12.5\%). According to the O-PNI, $72.5 \%$ of patients were considered to be high risk, and the median score was 36.5 (31.9-40.7). From evaluation by the CONUT, $25 \%$ of patients were considered severely malnourished, and the median score was $6.0(3.3-8.8)$.

\section{Relationship of Nutritional Status with Intestinal Resection and LOS}

Table 2 shows the relationship between nutritional status as determined by the various screening tools on admission and intestinal resection. There was tendency toward, but no significant relation between, intestinal re- section and SGA score, serum albumin level, total cholesterol level, O-PNI score, or CONUT score.

As shown in Table 3, significant differences in nutritional status were found between patients with LOS $\geq 28$ days (prolonged LOS group) and those with LOS $<28$ days (non-prolonged LOS group). When using the SGA or NRS 2002 for screening, malnutrition was significantly related to prolonged LOS. When using the MUST, there was a tendency toward, but no significant relation between, nutritional risk and prolonged LOS.

In addition, the nutritional parameters serum albumin, O-PNI score, CONUT score, and loss of body weight were correlated with LOS. To evaluate the prognostic yield, ROC curves were plotted for prolonged LOS (Fig. 1). As shown in Table 4, the cutoff values for serum albumin (sensitivity $57.1 \%$, specificity $89.5 \%$ ) and the OPNI (sensitivity $71.4 \%$, specificity $73.7 \%$ ) were classed as moderately accurate parameters.

By combining the serum albumin cutoff value and NRS 2002 score, patients were divided into 4 groups (Table 5). The prolonged LOS rate was $68.2 \%$ in the group with the worst prognosis.

\section{Discussion}

To our knowledge, this is the first study to report on the utility of several nutritional screening tools for hospitalized CD patients. We examined the usefulness of these nutritional tools from the viewpoint of intestinal resection rate and LOS. Our findings show that both the SGA and NRS 2002 were significantly related to prolonged LOS and are better screening tools than the MUST for CD patients.

Several studies have demonstrated a relationship between nutritional screening tools and prognosis. Stenvinkel et al. [17] reported that mild to moderate malnutrition, as assessed by the SGA, was present in 39\% of patients with end-stage renal disease and predicted outcome independently of age and comorbidity in both males and females. Furthermore, another report stated that SGA was an excellent predictor of outcome in patients undergoing liver transplantation [21]. Conversely, Tevik et al. [18] found that the incidence of complications and the median LOS were significantly higher in patients with NRS 2002 scores $\geq 3$, concluding that the NRS 2002 was a reliable screening tool in their sample of hospitalized patients with chronic heart failure. Moreover, multivariate analysis conducted by Tsaousi et al. [22] highlighted limited autonomy in everyday activities, MUST score, and 
Table 2. Relationship to nutritional status according to screening tools and intestinal resection

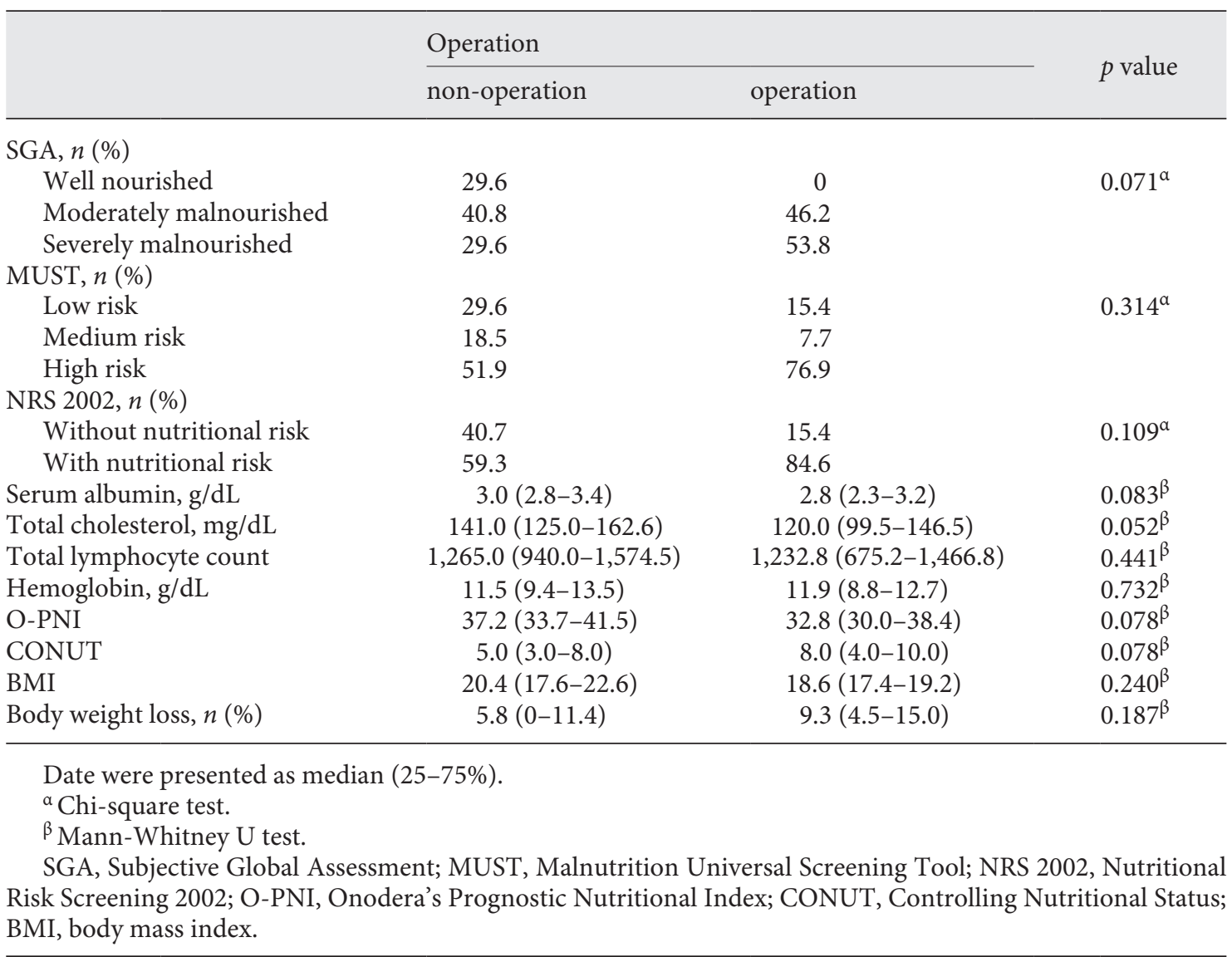

artificial nutrition as the most powerful predictors of pressure ulcers. Lomivorotov et al. [23] reported the prognostic value of different nutritional screening tools SGA, NRS 2002, MUST, Mini-Nutritional Assessment, and Short Nutritional Assessment Questionnaire - in patients undergoing cardiopulmonary bypass. In their study, both the MUST and Mini-Nutritional Assessment had independent predictive value with regard to postoperative complications. Our study, however, showed a significant relationship between LOS and the SGA or NRS 2002, but only a tendency toward a relation toward, and not a significant relation with, the MUST. Unlike the SGA and NRS 2002, the MUST does not assess changes in food intake. Given that many hospitalized CD patients have reduced intake, which results in loss of body weight, the MUST may not be a useful screening tool for CD patients. Furthermore, Csontos et al. [24] reported that the MUST did not provide sufficient information to enable selection and follow-up of potentially at-risk CD patients in outpatient care. Recently, Velasco et al. [25] used various nutri- tional screening tools to assess hospitalized patients and found relatively good agreement between the SGA, MUST, and NRS 2002. Not only the SGA and NRS 2002 but also the MUST need to be validated in larger samples of hospitalized CD patients by a multicenter study, because the number of $\mathrm{CD}$ patients from just a single hospital is limited in Japan.

Our results showed definite relationships between LOS and serum albumin, O-PNI, CONUT, and loss of body weight. Also, ROC analysis identified both serum albumin level and O-PNI score as good parameters for predicting prolonged LOS. Sachlova et al. [26] reported that the O-PNI provided significant prognostic value for survival in patients with metastatic or recurrent gastric cancer. In our study, in addition to serum albumin, the $\mathrm{O}-\mathrm{PNI}$ was a good predictor of prolonged LOS. The OPNI is calculated using the serum albumin level and total lymphocyte count. However, total lymphocyte count was not correlated with prolonged LOS in our study. Therefore, we surmise that the O-PNI and serum albumin are 
Table 3. Relationship to nutritional status according to screening tools and LOS

\begin{tabular}{|c|c|c|c|}
\hline & \multicolumn{2}{|l|}{ LOS } & \multirow{2}{*}{$p$ value } \\
\hline & $<28$ days $(n=21)$ & $\geq 28$ days $(n=19)$ & \\
\hline \multicolumn{4}{|l|}{ SGA, $n(\%)$} \\
\hline Well nourished & 38.1 & 0 & \multirow{3}{*}{$0.008^{\alpha}$} \\
\hline Moderately malnourished & 38.1 & 47.4 & \\
\hline Severely malnourished & 23.8 & 52.6 & \\
\hline \multicolumn{4}{|l|}{ MUST, $n(\%)$} \\
\hline Low risk & 38.1 & 10.5 & \multirow[t]{3}{*}{$0.058^{\alpha}$} \\
\hline Medium risk & 19.0 & 10.5 & \\
\hline High risk & 42.9 & 79.0 & \\
\hline \multicolumn{4}{|l|}{ NRS $2002, n(\%)$} \\
\hline Without nutritional risk & 47.6 & 15.8 & \multirow[t]{2}{*}{$0.032^{\alpha}$} \\
\hline With nutritional risk & 52.4 & 84.2 & \\
\hline Serum albumin, g/dL & $3.3(2.9-3.7)$ & $2.8(2.4-3.0)$ & $0.001^{\beta}$ \\
\hline Total cholesterol, mg/dL & $143.0(123.0-167.3)$ & $121.0(100.2-159.0)$ & $0.130^{\beta}$ \\
\hline Total lymphocyte count & $1,265.0(864.3-1,550.6)$ & $1,232.8(739.2-1,553.9)$ & $0.872^{\beta}$ \\
\hline Hemoglobin, g/dL & $11.7(10.2-13.7)$ & $11.2(8.8-12.4)$ & $0.093^{\beta}$ \\
\hline $\mathrm{O}-\mathrm{PNI}$ & $38.2(33.9-42.7)$ & $33.9(28.3-37.2)$ & $0.006^{\beta}$ \\
\hline CONUT & $5.0(2.5-7.5)$ & $7.0(5.0-10.0)$ & $0.019^{\beta}$ \\
\hline BMI & $20.4(17.8-22.5)$ & $18.9(16.7-22.1)$ & $0.215^{\beta}$ \\
\hline Body weight loss, $n(\%)$ & $4.2(0.0-9.9)$ & $11.0(5.0-13.0)$ & $0.044^{\beta}$ \\
\hline \multicolumn{4}{|c|}{$\begin{array}{l}\text { Date were presented as median (25-75\%). } \\
{ }^{\alpha} \text { Chi-square test. } \\
{ }^{\beta} \text { Mann-Whitney U test. } \\
\text { LOS, length of stay in the hospital; SGA, Subjective Global Assessment; MUST, Malnutrition Universal Scree- } \\
\text { ing Tool; NRS 2002, Nutritional Risk Screening 2002; O-PNI, Onodera's Prognostic Nutritional Index; CONUT, } \\
\text { ontrolling Nutritional Status; BMI, body mass index. }\end{array}$} \\
\hline
\end{tabular}

Table 4. Receiver operating characteristic analysis for factors affecting length of stay in the hospital

\begin{tabular}{lllllr}
\hline & AUC & $p$ value & Sensitivity & Specificity & Cutoff value \\
\hline Serum albumin, g/dL & 0.797 & 0.001 & 0.571 & 0.895 & 3.3 \\
O-PNI & 0.749 & 0.007 & 0.714 & 0.737 & 36.5 \\
CONUT & 0.716 & 0.082 & 0.737 & 0.619 & 5.5 \\
Body weight loss, \% & 0.687 & 0.044 & 0.895 & 0.476 & 3.7 \\
\hline
\end{tabular}

AUC, area under receiver operating characteristic curve; O-PNI, Onodera's Prognostic Nutritional Index; CONUT, Controlling Nutritional Status.

equivalent predictors. However, considering simplicity of screening, serum albumin is preferable for $\mathrm{CD}$ patients.

The significance of serum albumin as an indicator of nutritional status is controversial because the serum albumin level is affected by intravenous fluids and dehydration. Although our serum albumin levels did include data obtained from patients with dehydration or those transferred from the other hospital, our results showed a cor- relation between serum albumin levels and LOS. In line with our results, some studies have reported a relationship between serum albumin and prolonged LOS. El-Hussuna et al. [27] found that low preoperative albumin correlated with longer LOS in CD patients. Castillo et al. [28] reported that NRS 2002 score $>5$ and serum albumin $<2.5 \mathrm{~g} / \mathrm{dL}$ were the most significant variables associated with mortality and prolonged LOS. However, patients with various 


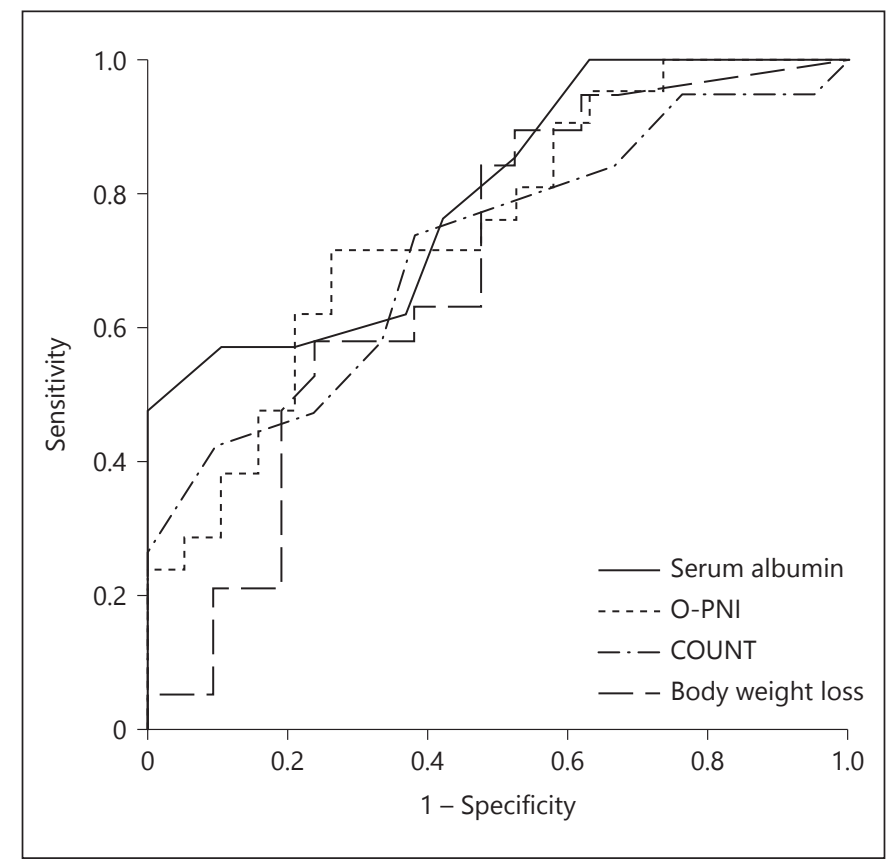

Fig. 1. Receiver operating characteristic curves for factors predicting prolonged length of hospital stay ( $\geq 28$ days). O-PNI, Onodera's Prognostic Nutritional Index; CONUT, Controlling Nutritional Status.

Table 5. Serum albumin and NRS 2002 enhance the prediction of prolonged LOS

\begin{tabular}{lll}
\hline \multirow{2}{*}{ Rate of prolonged LOS } & \multicolumn{2}{l}{ Serum albumin } \\
\cline { 2 - 3 } & $\leq 3.3 \mathrm{~g} / \mathrm{dL}$ & $>3.3 \mathrm{~g} / \mathrm{dL}$ \\
\hline $\begin{array}{l}\text { NRS 2002,\% }(n) \\
\text { Without nutritional risk } \\
\text { With nutritional risk }\end{array}$ & $11.1(1 / 9)$ & $50.0(2 / 4)$ \\
\hline
\end{tabular}

LOS, length of stay in the hospital.

diseases were enrolled in their study. In our study, the serum albumin cutoff value for prolonged LOS ( $\geq 28$ days) was $3.3 \mathrm{~g} / \mathrm{dL}$. We also investigated the relationship of serum albumin level with the SGA, MUST, and NRS 2002. We found that $53.8 \%$ of patients with serum albumin $<3.3$ $\mathrm{g} / \mathrm{dL}$ were categorized as well-nourished or moderately malnourished when using the SGA. When using the MUST, $30.8 \%$ of these patients were assessed as low risk or medium risk. On evaluation by the NRS 2002, only $15.4 \%$ of these patients were classified as being without nutritional risk (data not shown). Furthermore, examiner training may be necessary to improve competency before using SGA properly. NRS 2002 is more rapid and requires less examiner training than the SGA [25]. Taking into account the simplicity, a combination of serum albumin and NRS 2002 is very useful and an excellent nutritional manner for hospitalized CD patients. By combining the serum albumin cutoff value and the NRS 2002 score, the prolonged LOS rate was $68.2 \%$ in the group with the worst prognosis. From these results, NRS 2002 score and serum albumin level appear to be simple and useful predictors of prolonged LOS in hospitalized patients with CD.

Our study has limitations because of it being a single center study and of retrospective nature. First of all, the number of patients enrolled in this study is limited; hence, the significance of each parameter should be confirmed in the prospective multicenter study. Secondly, despite comparing LOS, the severity and treatment varies depending on the case. This is a small number study, and we suggest that further multicenter studies be carried out in the future to overcome these limitations.

\section{Conclusion}

The SGA and NRS 2002 were reliable screening tools for prolonged LOS in hospitalized patients with CD. In addition, serum albumin level and O-PNI score were good predictors of prolonged LOS. When taking simplicity of screening into account, serum albumin level is a better predictor of prolonged LOS in CD patients. We conclude that a combination of the NRS 2002 and serum albumin level may be useful for nutritional screening of hospitalized patients with CD.

\section{Disclosure Statement}

The authors declare no conflicts of interest.

References

Mayer L: Evolving paradigms in the pathogenesis of IBD. J Gastroenterol 2010;45:9-16.

-2 Andoh A, Kuzuoka H, Tsujikawa T, Nakamura S, Hirai F, Suzuki Y, Matsui T, Fujiyama Y, Matsumoto T: Multicenter analysis of fecal microbiota profiles in Japanese patients with Crohn's disease. J Gastroenterol 2012;47: 1298-1307.

-3 Andoh A, Imaeda H, Aomatsu T, Inatomi O, Bamba S, Sasaki M, Saito Y, Tsujikawa T, Fujiyama Y: Comparison of the fecal microbiota profiles between ulcerative colitis and Crohn's disease using terminal restriction fragment length polymorphism analysis. J Gastroenterol 2011;46:479-486. 
-4 Farmer RG, Hawk WA, Turnbull RB Jr: Clinical patterns in Crohn's disease: a statistical study of 615 cases. Gastroenterology 1975;68: 627-635.

-5 Mekhjian HS, Switz DM, Melnyk CS, Rankin GB, Brooks RK: Clinical features and natural history of Crohn's disease. Gastroenterology 1979;77:898-906.

6 Afonso JJ, Rombeau JL: Nutritional care for patients with Crohn's disease. Hepatogastroenterology 1990;37:32-41.

7 Driscoll RH Jr, Rosenberg IH: Total parenteral nutrition in inflammatory bowel disease. Med Clin North Am 1978;62:185-201.

8 Takaoka A, Sasaki M, Kurihara M, Iwakawa $\mathrm{H}$, Inoue $\mathrm{M}, \mathrm{Bamba} \mathrm{S}$, Ban $\mathrm{H}$, Andoh A, Miyazaki Y: Comparison of energy metabolism and nutritional status of hospitalized patients with Crohn's disease and those with ulcerative colitis. J Clin Biochem Nutr 2015;56: 208-214.

9 Baker JP, Detsky AS, Wesson DE, Wolman SL, Stewart S, Whitewell J, Langer B, Jeejeebhoy KN: Nutritional assessment: a comparison of clinical judgement and objective measurements. N Engl J Med 1982;306:969-972.

$>10$ Detsky AS, Baker JP, Mendelson RA, Wolman SL, Wesson DE, Jeejeebhoy KN: Evaluating the accuracy of nutritional assessment techniques applied to hospitalized patients: methodology and comparisons. JPEN J Parenter Enteral Nutr 1984;8:153-159.

$>11$ Sungurtekin H, Sungurtekin U, Hanci V, Erdem E: Comparison of two nutrition assessment techniques in hospitalized patients. $\mathrm{Nu}$ trition 2004;20:428-432.

$\checkmark 12$ Kondrup J, Allison SP, Elia M, Vellas B, Plauth M: ESPEN guidelines for nutrition screening 2002. Clin Nutr 2003;22:415-421.

13 Stratton RJ, Hackston A, Longmore D, Dixon $\mathrm{R}$, Price S, Stroud M, King C, Elia M: Malnu- trition in hospital outpatients and inpatients: prevalence, concurrent validity and ease of use of the 'malnutrition universal screening tool' ('MUST') for adults. Br J Nutr 2004;92: 799-808.

14 Kondrup J, Rasmussen HH, Hamberg O, Stanga Z: Nutritional risk screening (NRS 2002): a new method based on an analysis of controlled clinical trials. Clin Nutr 2003;22: 321-336.

15 Onodera T, Goseki N, Kosaki G: Prognostic nutritional index in gastrointestinal surgery of malnourished cancer patients. Nihon Geka Gakkai Zasshi 1984;85:1001-1005.

16 Ignacio de Ulíbarri J, González-Madroño A, de Villar NG, González P, González B, Mancha A, Rodriguez F, Fernandez G: CONUT: a tool for controlling nutritional status. First validation in a hospital population. Nutr Hosp 2005;20:38-45.

17 Stenvinkel P, Barany P, Chung SH, Lindholm B, Heimbürger O: A comparative analysis of nutritional parameters as predictors of outcome in male and female ESRD patients. Nephrol Dial Transplant 2002;17:1266-1274.

18 Tevik K, Thürmer H, Husby MI, de Soysa AK, Helvik AS: Nutritional risk screening in hospitalized patients with heart failure. Clin Nutr 2015;34:257-264.

19 Best WR, Becktel JM, Singleton JW, Kern F Jr: Development of a Crohn's disease activity index. National Cooperative Crohn's Disease Study. Gastroenterology 1976;70:439-444.

20 Best WR, Becktel JM, Singleton JW: Rederived values of the eight coefficients of the Crohn's Disease Activity Index (CDAI). Gastroenterology 1979;77:843-846.

21 Stephenson GR, Moretti EW, El-Moalem H, Clavien PA, Tuttle-Newhall JE: Malnutrition in liver transplant patients: preoperative subjective global assessment is predictive of out- come after liver transplantation. Transplantation 2001;72:666-670.

22 Tsaousi G, Stavrou G, Ioannidis A, Salonikidis S, Kotzampassi K: Pressure ulcers and malnutrition: results from a snapshot sampling in a university hospital. Med Princ Pract 2015;24:11-16.

23 Lomivorotov VV, Efremov SM, Boboshko VA, Nikolaev DA, Vedernikov PE, Deryagin MN, Lomivorotov VN, Karaskov AM: Prognostic value of nutritional screening tools for patients scheduled for cardiac surgery. Interact Cardiovasc Thorac Surg 2013;16:612-618.

24 Csontos ÁA, Molnár A, Piri Z, Pálfi E, Miheller P: Malnutrition risk questionnaire combined with body composition measurement in malnutrition screening in inflammatory bowel disease. Rev Esp Enferm Dig 2017; 109:26-32.

-25 Velasco C, García E, Rodríguez V, Frias L, Garriga R, Alvarez J, Garcia-Peris P, Leon M: Comparison of four nutritional screening tools to detect nutritional risk in hospitalized patients: a multicentre study. Eur J Clin Nutr 2011;65:269-274.

26 Sachlova M, Majek O, Tucek S: Prognostic value of scores based on malnutrition or systemic inflammatory response in patients with metastatic or recurrent gastric cancer. Nutr Cancer 2014;66:1362-1370.

27 El-Hussuna A, Iesalnieks I, Horesh N, Hadi S, Dreznik Y, Zmora O: The effect of pre-operative optimization on post-operative outcome in Crohn's disease resections. Int J Colorectal Dis 2017;32:49-56.

28 Castillo Pineda JC, Gómez García A, Velasco N, Díaz-Pizarro Graf JI, Matos Adámes A, Miján de la Torre A: Nutritional assessment of hospitalized patients in Latin America: association with prognostic variables. The ENHOLA study. Nutr Hosp 2016;33:275. 